\title{
EVALUATING THE PERFORMANCE OF A DAYLIGHTING TRADITIONAL DEVICE, THE MASHRABIYA, IN CLEAR SKY CONDITIONS: CASE STUDY OF A TRADITIONAL BAHRAINI HOUSE
}

\author{
HANA M. ALJAWDER ${ }^{1} \&$ HALA A. EL-WAKEEL ${ }^{2}$ \\ ${ }^{1}$ Department of Architecture \& Interior Design, College of Engineering, University of Bahrain, Bahrain \\ ${ }^{2}$ Interior Design Department, College of Design, Imam Abdulrahman Bin Faisal University, Saudi Arabia
}

\begin{abstract}
The mashrabiya is used in traditional Islamic architecture to provide visual privacy, reduce the glare from direct sunlight, and allow natural ventilation. Traditional Bahraini houses were designed with specific features and characteristics to cater to the cultural and religious needs of the occupants and the bio-climatic conditions. This study has investigated the impact of the mashrabiya on daylight performance within a space and examined how it affects the quantity and quality of the daylight that is admitted into the space. A field study was conducted in one of the traditional houses in Bahrain for evaluating the performance of the mashrabiya in providing daylight in the space. The studied room was located on the upper floor and had an $\mathrm{L}$ shape layout $\left(27.75 \mathrm{~m}^{2}\right)$. The main wall of this room faced the north with openings covered with mashrabiya, while the inner part of the room (the south wall) overlooked an atrium with a skylight. The south wall had two small openings that increased the illumination level in a small part of the room. The results showed that the performance of daylight throughout the mashrabiya was better, to some extent, than without any screen. The mashrabiya in this case can provide the level of illuminance recommended for residential activities and can provide better uniformity as compared to the space without a mashrabiya. The mashrabiya device is capable of reducing the impact of glare in the whole space. It is preferable to improve the design of the mashrabiya to enhance the uniformity of daylight in the space. The chromaticity of light in the space, as the CCT calculation for all the measured points below the accepted level, was another challenge with regards to the performance of the mashrabiya device. A mashrabiya is likely to demonstrate a more successful performance and better daylighting if it is designed and modified to consider uniformity and the chromaticity of light in the space.
\end{abstract}

Keywords: Bahrain, day lighting, illuminance, mashrabiya, traditional device.

\section{INTRODUCTION}

The mashrabiya is a device used in traditional Islamic architecture for several reasons, such as to provide visual privacy, reduce the glare from direct sunlight, and allow natural ventilation [1]-[3]. Privacy and bio-climatic comfort are perhaps the most important influences on older residential designs in Bahrain. Traditional Bahraini houses were designed with specific features and characteristics to cater to the cultural and religious needs of the occupants and the bio-climatic conditions. Victor Olgyay [4] has initially used the term bio-climatic by developing a bio-climatic chart to study the climatic data for thermal comfort limits and for identifying design strategies. The presence of bio-climatic conditions has shown sustainability since periods in the native conventional settlements [5]. The climate-sensitive passive design and other adaptive features of conventional architecture and construction methods are emphasized in other countries with hot climates with respect to their bio-climatic conditions [6].

Recently, construction practices are changed due to the availability of modern standardized building methods that dominate the country's market. The impact of electronic media and market economy has changed the design of architecture even in rural, semi-rural, 
and remote areas along with a change in socio-cultural outlook. The modern construction practices are usually conducted without giving substantial considerations to the local cultural and bio-climatic conditions, which may have implications on energy consumption to sustain comfortable living conditions inside the houses. the inherent logic of the traditional practices. A comparative study between native and present-day constructions can be revealed.

These houses often consist of a central courtyard surrounded by rooms (Fig. 1). The courtyard is open to the sky and all the rooms have windows facing towards it, which allows daylight to enter as well as permits cross-ventilation through the rooms. The privacy has been ensured through few openings provided in the external facade (Fig. 2). The external windows are usually divided into two parts: the higher parts are covered with stained or transparent glass and the lower parts are usually covered with the mashrabiya. This division of windows allows for ventilation and a view outside without compromising privacy.

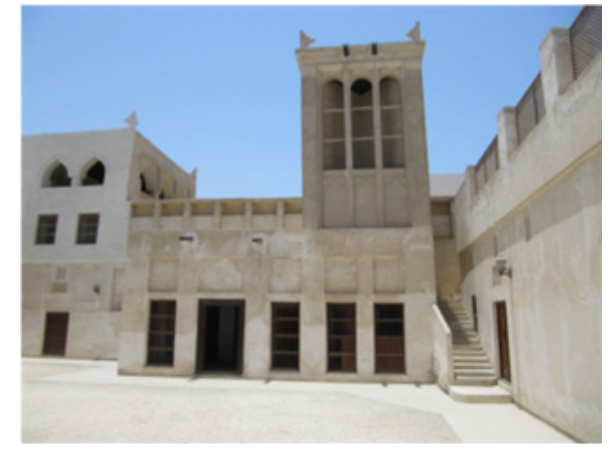

Figure 1: An example of a traditional Bahraini house with a central courtyard.

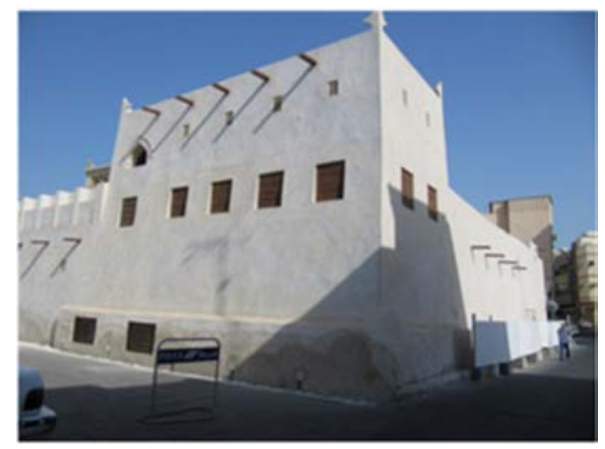

Figure 2: An example of the external facade of a traditional Bahraini house.

Bahrain has a very destructive and severe climate as it is located with high humidity in a hot arid region and minimal diurnal range. The major objective of the courtyard was to safeguard the resident from that climate. By offering internal shade during the day for activating air flow in the house, the apparent sky conditions assist to release part of the stored heat by radiation to the sky even though it is humid. The courtyard house and the mashrabiya concepts are complemented by the local builder to invent different cooling and ventilating methods of the house. 
The dimension of the plan is lower than the height of the courtyard in traditional houses in Bahrain, with a proportion of 1:1.5. Therefore, the total area is reduced to sun and it offers appropriate shade within the house. This thermal property is proven several surveys on courtyard houses. In a traditional house, the difference of $5^{\circ} \mathrm{C}$ was observed between the temperature on the roof and the temperature in the courtyard, and $11^{\circ} \mathrm{C}$ between inside and outside house rooms. The owner is encouraged by the defined space of the courtyard to plant trees and vegetation throughout his private territory. The microclimate condition is regulated by landscaping to offer visual and shade pleasure throughout the adverse house environment. In adaptive building skins, the development and potential integration of hygromorphic materials offers benefits for designing passively responsive bioclimatic architecture that is in consistent synchronization with ambient moisture and variable levels of atmospheric humidity. The extreme climatic conditions are converted by the traditional architecture in Bahrain into an appropriate local construction culture that estimates the modern bioclimatic approach for designing.

\subsection{Background of Bahrain}

Bahrain is a small archipelago consisting of 35 islands. Bahrain is located to the north of the Tropic of Cancer. It is $757.50 \mathrm{~km}^{2}$ in area and located in the Arab Gulf at a latitude of $26^{\circ}$ $16^{\prime} \mathrm{N}$ and a longitude of $50^{\circ} 39^{\prime} \mathrm{E}$ [7]. Bahrain is a hot, humid country where the mean maximum temperature is $42^{\circ} \mathrm{C}$ in July and the mean minimum temperature is $10.3^{\circ} \mathrm{C}$ in January (Bahrain International Airport website). The annual average relative humidity in Bahrain is $65 \%$, with a maximum average of $77 \%$ in December and minimum average of $50 \%$ in June [8]. It has clear sky conditions and plenty of sunlight throughout the year. The maximum average daily hours of sunshine (daylight hours) is 11.3 hours in June and the minimum is 7.3 hours in January [9]. The mean daylight global horizontal illuminance is $50,000 \mathrm{~lx}[10]$.

\section{THE CASE STUDY}

Al-Zayed's house belonged to Abdullah bin Ali Al-Zayed (1899-1945) and is considered to be of cultural significance in Bahrain. Therefore, Sheikh Mai Al-Khalifa, the Minister of Culture, has taken on the responsibility of caring for these types of houses since 2002, before she became the Minister of Culture in 2008. Al-Zayed's house was built 100 years ago; however, it was opened to the public in 2003 after renovation [11]. The house consists of two storeys and has an internal courtyard (Figs 3 and 4). The main facade faces north (Fig. 5). The studied room is located on the upper floor and has an L shape $(27.75 \mathrm{~m} 2)$. The main wall of this room faces north and has openings covered with mashrabiya. The inner part of the room (the south wall) overlooks an atrium with a skylight. This south wall has two small openings that increase the illumination level in a small part of the room (Area 2 in Fig. 4). This obviously affects the analysis of the results for the whole room. Therefore, it was decided to divide the room into two zones and to focus on the zone that was close to the opening covered with mashrabiya to obtain accurate results in the analysis of the mashrabiya's performance (Area 1 in Fig. 4). The area of the studied zone is $18.6 \mathrm{~m}^{2}$.

The present study has investigated the effect of the mashrabiya on daylight performance in a space. It aims to study the mashrabiya's characteristics and to examine how it affects the quantity and quality of the daylight that is admitted into the space. The performance of several shading systems was evaluated through simulation software and physical models to determine daylight uniformity distribution and daylight levels throughout the space. The 


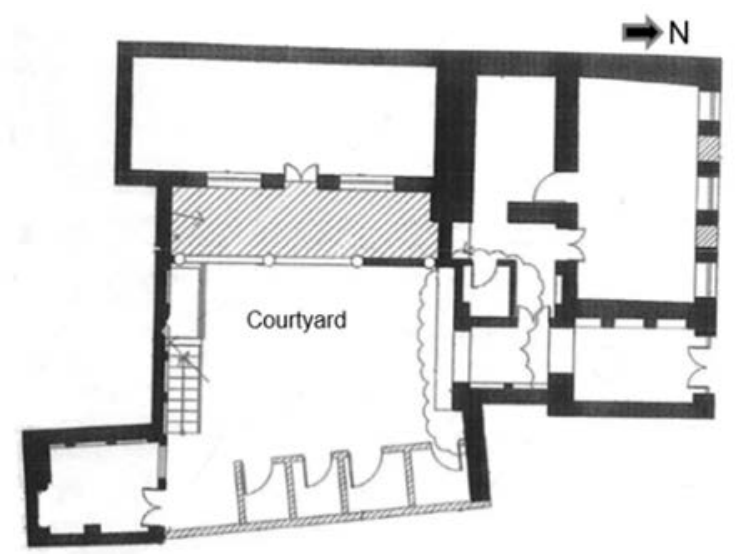

Figure 3: Ground floor plan for Abdullah Al-Zayed's house [12].

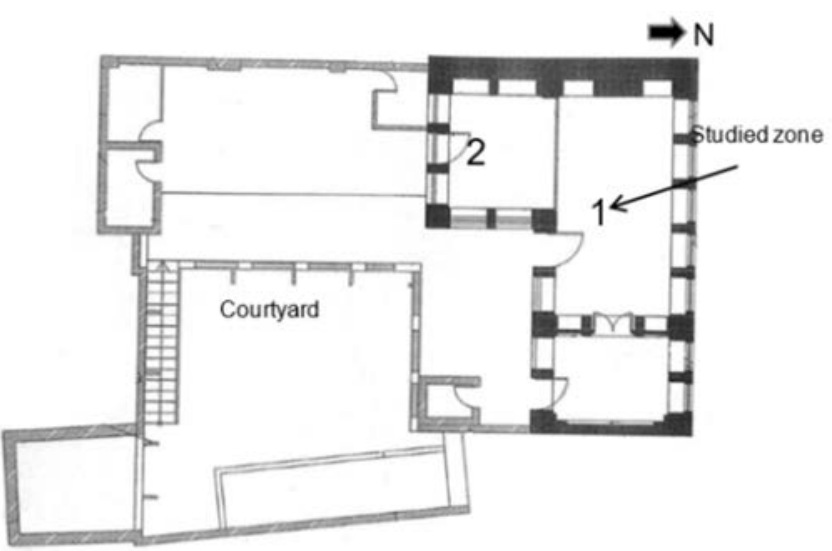

Figure 4: First floor plan for Abdullah Al-Zayed's house [12].

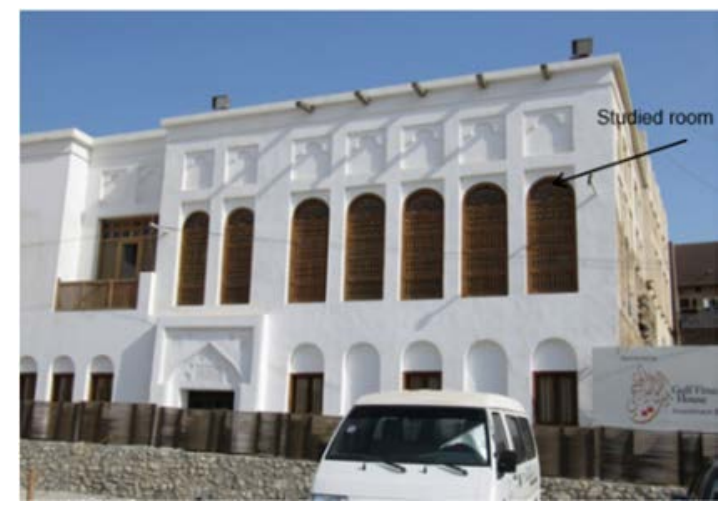

Figure 5: North facade of Al-Zayed's house showing the mashrabiya screen. 
perforation percentages were recommended by other studies to use simulations based on the illumination levels offered by the mashrabiya; a daylight and visual privacy device), for increasing the illuminance level. In contrast, the paucity of studies toward the assessment of the performance of the current well-established traditional device enables the researcher to study the daylight uniformity distribution in controlling the glare throughout the residential space. Additionally, the performance of the mashrabiya was compared with a similar condition in the room regardless of using any device. Thereby, this study has intended to evaluate the aforementioned unidentified elements by studying the current house using a mashrabiya device in the context of Bahrain. The study has comprehensively focused on the uniformity of daylight and the illuminance in the space, and the control of glare, which were not researched due to the lack of literature. Lastly, the study has compared the performance of both situations through the simulation of similar study space without using the mashrabiya.

\section{MATERIAL AND METHODS}

Surveys of some traditional houses in Bahrain were made to choose a suitable house for the case study. Al-Zayed's house was selected and permission was obtained from the Ministry of Culture to conduct this study. This part of the research work was divided into two stages. The first stage involved measurements of daylight in the house of Abdullah Al-Zayed to measure and analyse the illuminance through traditional window devices (the mashrabiya) at specific dates and times to determine the impact of the mashrabiya on daylight in the space. In the second stage, simulation software Ecotect Analysis and Desktop Radiance were used and a similar room without a mashrabiya was evaluated.

\subsection{Instrument/apparatus}

The instruments were fixed on the top of the tripod to obtain accurate readings in both a horizontal and a vertical position. A digital illuminance meter (Konica Minolta T-10) was used to measure the daylight level inside and outside of the room. A digital chromaticity meter (Konica Minolta Chroma Meter CL-100) was also used to measure the chromaticity of daylight inside the room. A Portable Spectrophotometer CM-2600d (Konica Minolta) was used to measure the reflectance of the surfaces. Finally, a tripod was used to take the readings at $0.80 \mathrm{~m}$. The measurements were taken at a height of $0.80 \mathrm{~m}$ because the people who occupied the house at that time used to sit on a cushion at floor level, which put their eye level at approximately $0.80 \mathrm{~m}$ above the floor. Further, $0.80 \mathrm{~m}$ is the height used to accomplish many daily tasks (e.g., preparing food on a surface, reading on a desk, etc.).

\subsection{Measurements}

Visits were carried out to take all the measurements of the room and window openings. A floor plan drawing with $1 \mathrm{~m}^{2}$ grids for the room was prepared to locate the readings, which were measured by the photometric and colorimetric instruments (Fig. 6). The readings were taken in one sequence starting from point 1 at the forward left corner to point 28 at the rear right corner of the room. Illuminance and chromaticity readings were taken in the selected room in both summer (from 11-26 July 2012) and winter (from 22 December to 23 January 2013). Five readings were taken for each point: the first was horizontal and the second, third, fourth and fifth were vertical readings starting parallel with the mashrabiya at $0^{\circ}, 90^{\circ}, 180^{\circ}$, and $270^{\circ}$ clockwise, respectively. The readings were taken for each point at three times during the day (9:00 am, solar noon, and 3:00 pm). All readings were taken at two levels: floor level and $0.80 \mathrm{~m}$ above the floor. Furthermore, the measurements of the illuminance 
and chromaticity were taken separately on different days to ensure the readings were recorded at the same time (9:00 am, solar noon, and 3:00 pm) for each measurement. A similar drawing with the same grid was also prepared to measure the illuminance without the mashrabiya using the simulation software.

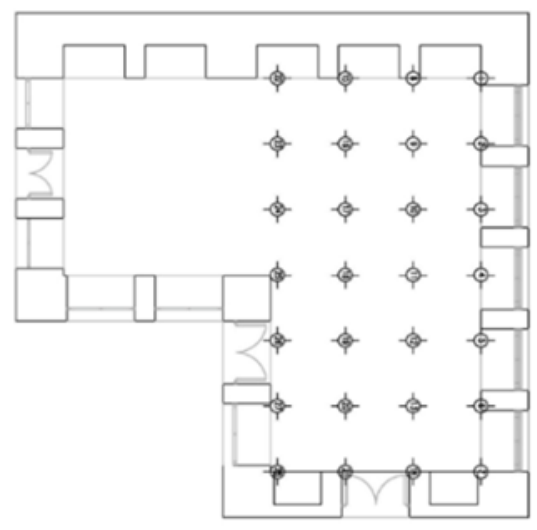

Figure 6: Floor plan of the studied room in Al-Zayed's house showing the measurement points.

\subsection{Simulation}

Ecotect Analysis software is being used by the designer in the basic stages of a design to carry out primary analysis for the design projects [13]-[14]. This helped in modelling the space being studied or to import the model from AutoCAD, Google SketchUp, or Rhino. In the present study, the model was exported to Desktop Radiance to carry out the lighting analysis, after modelling the building and specifying all the above-mentioned parameters in Ecotect. In addition, the date, time, and sky conditions were also specified in Radiance to obtain accurate results. Fig. 7 has illustrated the sun path diagram of the location of the studied house using Ecotect analysis software.

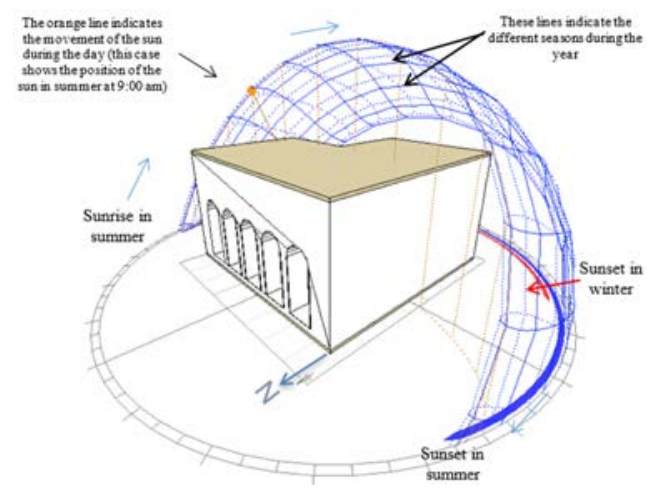

Figure 7: Annual sun path diagram and north direction for the studied room in Al-Zayed's house (Prepared using Ecotect Analysis software). 


\subsection{Characterization of the mashrabiya}

The studied room of this house was located on the second floor. The openings of this room faced north and were covered with five equal-sized traditional screens (mashrabiya) (Fig. 8). Each window opening covered with mashrabiya was $175 \mathrm{~cm}$ in height, $82 \mathrm{~cm}$ in width, and $20 \mathrm{~mm}$ in depth. They were of a brown colour with $34.7 \%$ reflectance and the perforation ratio of the mashrabiya was $22 \%$. This percentage was calculated using a photograph taken of the mashrabiya with light passing through the openings. This created a strong contrast between the brown wooden part of the mashrabiya and the openings. The Fiji Image J program was used to count the pixels of the lighter areas, which helped to calculate the percentage of the irregular-shaped openings.

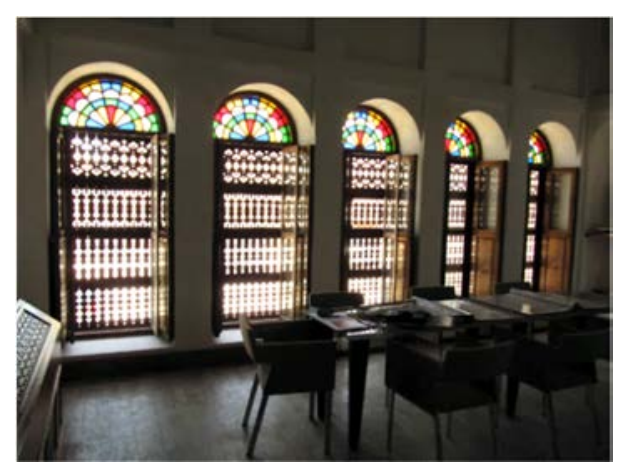

Figure 8: The five equal openings of the mashrabiya at the studied room of Al-Zayed house.

\subsection{Analysis of actual and computer-based daylight simulation}

The daylight analysis in this study helped in better understanding of the performance of the mashrabiya in admitting daylight into the space. Through this study, the average illumination level in the room with and without mashrabiya was analysed. This was then compared with the recommended average illumination level required in houses. The uniformity of the daylight distribution in the space was analysed for both situations with and without the mashrabiya. The chromaticity of light within the space was studied and the influence of the mashrabiya on the chromaticity of the daylight was evaluated. Finally, the effect of glare was quantified using the simplified daylight glare probability (DGPs).

\section{RESULTS AND DISCUSSION}

\subsection{Measurement}

\subsubsection{Average Illuminance}

The mean illuminance was calculated as illustrated in eqn (1):

$$
E_{\text {avg }}=\frac{\sum_{i=1}^{N} E_{i}}{N},
$$

where: $E_{a v g}=$ is the average illuminance (lx); $E_{i}=$ is the illuminance for each point (lx); $N=$ is the number of measurement points. 
Additionally, a calculation of the blocked daylight through the mashrabiya showed that the mashrabiya blocked $88.6 \%$ and $85 \%$ of the daylight in summer and winter, respectively. Eqn (2) was used to calculate the blocked light

$$
\text { Blocked light } \%=\frac{\left(E_{\text {avg without mashrabiya }}-E_{\text {avg with mashrabiya })}\right.}{E_{\text {avg without mashrabiya }}} * 100 \%,
$$

where $E_{\text {avg }}$ is the average illuminance (lx)

4.1.2 Daylight uniformity ratio

The uniformity of daylight distribution in the space is to calculate the minimum illuminance to average illuminance ratio (Emin/Eavg). The ratio can be calculated by using equation (3):

$$
\text { Uniformity Ratio }=E_{\min } / E_{\text {avg }} \text {, }
$$

where: $E_{\min }$ is the minimum illuminance in the space (lx); $E_{\text {avg }}$ is the average illuminance (lx).

The results were found to be very close to the recommended ratio 0.4 . Table 1 presents the uniformity ratios for the room with the mashrabiya in summer and winter.

Table 1: Average illuminance (lx), uniformity ratio in summer and winter with mashrabiya.

\begin{tabular}{|c|c|}
\hline $\begin{array}{l}\text { Average illuminance } \\
\text { with mashrabiya (lx) }\end{array}$ & Time \\
\hline \multicolumn{2}{|c|}{ Winter } \\
\hline 235 & 9:00 AM \\
\hline 218 & Solar noon \\
\hline $193 \quad$ Summer \\
\hline \multicolumn{2}{|c|}{ 3:00 PM } \\
\hline 15900 AM \\
\hline 99 & Solar noon \\
\hline $\begin{array}{c}\text { Uniformity Ratio } \\
\text { with mashrabiya (lx) }\end{array}$ & Time \\
\hline \multicolumn{2}{|c|}{ Winter } \\
\hline 0.4 & 9:00 AM \\
\hline 0.5 & Solar noon \\
\hline 0.4 & $3: 00$ PM \\
\hline \multicolumn{2}{|c|}{ Summer } \\
\hline 0.4 & 9:00 AM \\
\hline 0.4 & Solar noon \\
\hline
\end{tabular}

\subsubsection{Chromaticity of lighting}

The correlated colour temperature (CCT) was calculated from the CIE 1931 (x, y) measured in the space using the same points. Table 2 has shown the average CCT and illuminance for summer and winter at three different times of the day (9:00 am, noon, and 3:00 pm). 
Table 2: Average CCT and average illuminance for summer and winter.

\begin{tabular}{|c|c|c|c|c|}
\hline Time & \multicolumn{2}{|c|}{ Average CCT $(\mathrm{K})$} & \multicolumn{2}{c|}{ Average illuminance with mashrabiya in lx } \\
\hline & Winter & Summer & Winter & Summer \\
\hline 9:00 am & 4541 & 4351 & 235 & 159 \\
\hline Solar noon & 4303 & 4339 & 218 & 180 \\
\hline 3:00 pm & 4390 & 4212 & 193 & 99 \\
\hline
\end{tabular}

The average CCT values are very close for all times in both summer and winter. These values indicate the intermediate temperatures of the colour appearance as warm $<3300 \mathrm{~K}$ and cold $>5300$ (AS/NZS1680.1, 1990). Referring to the Kruithof curve (Fig. 9 and 10), all the values of the average CCT and average illuminance for summer and winter are located within the "uncomfortable" zone.

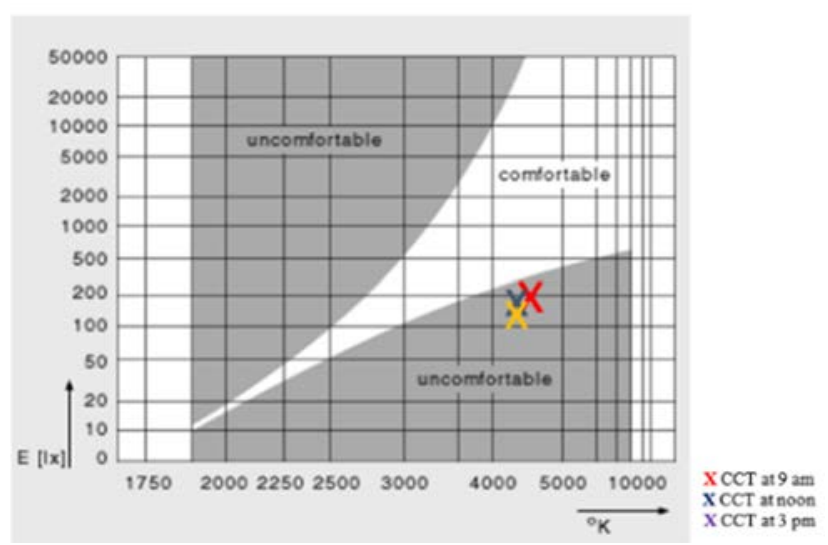

Figure 9: Kruithof curve with the average value of CCT and average illuminance for the studied room during summer [15].

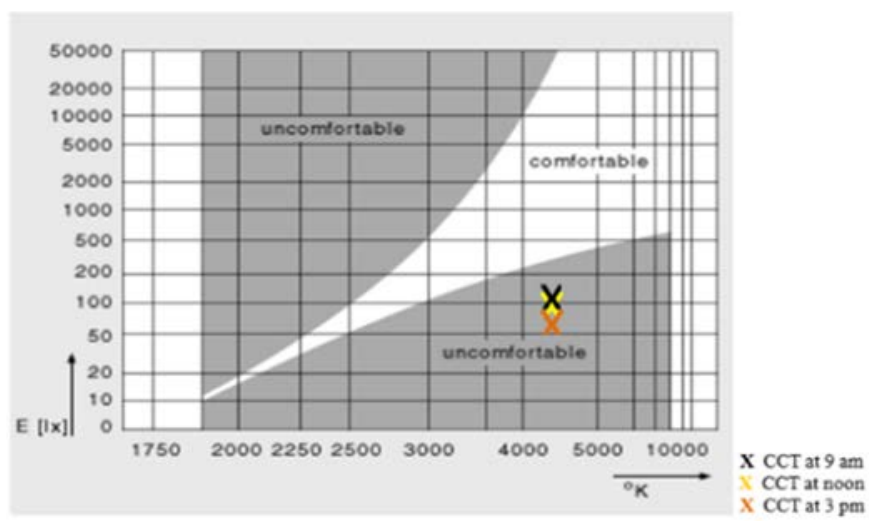

Figure 10: Kruithof curve of the average values of CCT and average illuminance for the studied room during winter [15]. 
4.1.4 Daylight glare probability index

A simplified daylight glare index (DGPs) was calculated for each point in the studied space for the specified times and seasons (Tables 3-8) using eqn (4) [16], [17]:

$$
D G P_{s}=\left(6.22 \times 10^{-5} \times E_{v}\right)+0.184,
$$

where $E_{v}$ is the vertical illuminance at eye level (lx). The vertical illuminance was measured at $0.8 \mathrm{~m}$ as people who occupied traditional houses in Bahrain used to sit on the floor.

Table 3: Simplified daylight glare probability (DGPs) for summer at 9:00 am, noon, and 3:00 pm.

\begin{tabular}{|c|c|c|c|c|c|c|c|c|c|c|c|c|c|c|c|c|c|c|c|c|c|c|c|c|c|c|c|}
\hline ints & & & & 3 & 4 & 5 & 6 & & 8 & 9 & & 11 & & & 14 & 15 & 16 & 17 & \begin{tabular}{l|l|l}
18 & 19 \\
\end{tabular} & $\begin{array}{l}920 \\
\end{array}$ & 212 & 22 & \begin{tabular}{ll|l}
3 & 24 \\
\end{tabular} & 25 & 26 & 27 & 2 \\
\hline $\mathrm{v}$ (lx) & & & $\tilde{m}$ & త్ & in & $\mid \begin{array}{l}: \\
: \\
\stackrel{\circ}{2}\end{array}$ & i & $\sim \mid$ & $\mid \begin{array}{l}0 \\
0 \\
0 \\
0\end{array}$ & & స్ & ๕ & ț & $\Xi$ & है & \begin{tabular}{|l|l}
\multirow{f}{*}{} \\
\end{tabular} & $\begin{array}{l}0 \\
0\end{array}$ & \begin{tabular}{l}
8 \\
\multirow{7}{*}{} \\
\end{tabular} & \begin{tabular}{l}
$\stackrel{f}{f}$ \\
\multirow{f}{*}{}
\end{tabular} & $\tilde{\sigma}$ & 亲 & $\stackrel{n}{\check{r}}$ & $\begin{array}{lll}0 \\
f \\
f\end{array}$ & \begin{tabular}{|c|c|}
$\infty$ \\
$\dot{\sigma}$ \\
\end{tabular} & in & à & 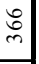 \\
\hline & & 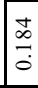 & ণ্ণ & ల్ల & f & \begin{tabular}{|l|l} 
\\
0 \\
0 \\
0
\end{tabular} & 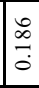 & $\mid$ & 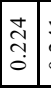 & $\underset{\sim}{\vec{f}}$ & $\vec{d}$ & $\underset{J}{*}$ & 㠻 & 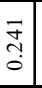 & 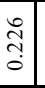 & \begin{tabular}{|l|}
\multirow{2}{*}{} \\
\\
\end{tabular} & 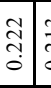 & $\begin{array}{l}m \\
\stackrel{m}{0} \\
0\end{array}$ & 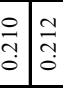 & 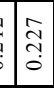 & \begin{tabular}{|c|}
\multirow{2}{*}{} \\
$\stackrel{1}{0}$ \\
\end{tabular} & 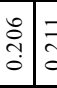 & 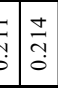 & 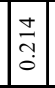 & तิ & $\begin{array}{l} \\
\\
\vdots \\
0\end{array}$ & \\
\hline & & & 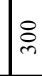 & i. & ปั & 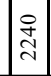 & 5 & $\approx$ & $\stackrel{2}{2}$ & 衣 & $\overrightarrow{0}$ & $\bar{\sigma}$ & $\hat{\alpha}$ & $\grave{n}$ & ì & 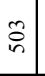 & $\frac{n}{6}$ & $\overline{\mathcal{F}}$ & 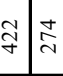 & f & 妾 & $\stackrel{n}{m} 7$ & $=$ & $\mid \begin{array}{l}\infty \\
\infty \\
\infty\end{array}$ & $\widetilde{\gamma}$ & $\begin{array}{l}8 \\
\vdots \\
+ \\
\end{array}$ & m \\
\hline & & & ฯ & $m$ & है & 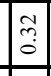 & $\frac{2}{0}$ & 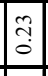 & \begin{tabular}{|l|}
\multirow{2}{*}{} \\
\multirow{2}{*}{} \\
\end{tabular} & \begin{tabular}{l}
\multirow{2}{*}{} \\
$\stackrel{0}{0}$ \\
\end{tabular} & \begin{tabular}{l|l}
\multirow{2}{*}{} & \\
& \\
\end{tabular} & 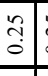 & $\begin{array}{l}n \\
\\
0 \\
0\end{array}$ & 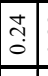 & $\begin{array}{l}\tilde{y} \\
\tilde{\theta} \\
0\end{array}$ & \begin{tabular}{|l|}
\multirow{2}{*}{} \\
$\stackrel{1}{\circ}$
\end{tabular} & \begin{tabular}{l|l}
\multirow{2}{*}{} & \\
& \\
\end{tabular} & \begin{tabular}{|l|}
$\vec{r}$ \\
$\vdots$ \\
\end{tabular} & 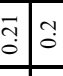 & \begin{tabular}{|l|} 
\\
\multirow{2}{*}{} \\
\hdashline \\
\end{tabular} & \begin{tabular}{|l}
$\vec{\tau}$ \\
$\stackrel{\circ}{\circ}$
\end{tabular} & \begin{tabular}{l|l}
$\vec{s}$ & $\overrightarrow{0}$ \\
& 0 \\
\end{tabular} & \begin{tabular}{|l|l}
$\bar{y}$ & $\vec{\Im}$ \\
\end{tabular} & \begin{tabular}{|l|}
$\overrightarrow{0}$ \\
\end{tabular} & \begin{tabular}{l|l}
$\vec{y}$ \\
\\
\end{tabular} & \begin{tabular}{|l|}
$\vec{y}$ \\
0 \\
\end{tabular} & z \\
\hline & & ; & & ปี & $\infty$ & 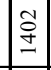 & in & लै & $\vec{F}$ & $\stackrel{\circ}{\circ}$ & $:$ & $\circ$ & $\stackrel{2}{\Omega}$ & 중 & $\begin{array}{l}\infty \\
b \\
b\end{array}$ & $\stackrel{\check{m}}{2}$ & \begin{tabular}{l|l}
+ \\
$\dot{\sigma}$
\end{tabular} & $\stackrel{\odot}{7}$ & $\vec{m} \frac{m}{m}$ & $\hat{n}$ & in & & î & f & t్ర & $\vec{\infty}$ & \\
\hline & & & & & & \begin{tabular}{|l|}
$\overrightarrow{\widehat{T}}$ \\
$\stackrel{0}{0}$
\end{tabular} & & & \begin{tabular}{|l|}
$\overrightarrow{\vec{y}}$ \\
\multirow{2}{*}{} \\
\end{tabular} & \begin{tabular}{|c|}
$\overrightarrow{\tilde{n}}$ \\
$\dot{0}$ \\
\end{tabular} & & & & & & $\stackrel{\infty}{\circ}$ & 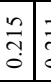 & \begin{tabular}{l|l}
$\overrightarrow{\vec{T}}$ & \\
&
\end{tabular} & & 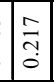 & 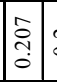 & & 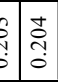 & & 0 & 1 & \\
\hline
\end{tabular}

Table 4: Simplified daylight glare probability (DGPs) for winter at 9:00 am, noon, 3:00 pm.

\begin{tabular}{|c|c|c|c|c|c|c|c|c|c|c|c|c|c|c|c|c|c|c|c|c|c|c|c|c|c|c|c|c|c|}
\hline $\begin{array}{l}\text { Measurement } \\
\text { points }\end{array}$ & & 1 & 2 & 3 & 4 & 5 & 6 & 7 & 8 & 9 & 10 & 11 & 12 & 13 & 14 & 15 & 16 & 17 & 18 & 19 & 20 & 21 & 22 & 23 & 24 & 25 & 26 & 27 & 28 \\
\hline Ev (lx) & \multirow{2}{*}{$\mid \begin{array}{l}\sum \\
\sum \\
0 \\
0\end{array}$} & 0 & $\because$ & ชิ & 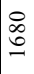 & 苟 & 0 & $ㅇ$ & $\begin{array}{l}0 \\
i\end{array}$ & $\tilde{\infty}$ & $\stackrel{\infty}{\infty}$ & 공 & $\stackrel{\tilde{o}}{0}$ & $\underset{\infty}{\stackrel{\infty}{\infty}}$ & $\hat{\sigma}$ & ĩ & $\underset{\widetilde{ర}}{\infty}$ & ‡ & $\hat{m}$ & $\frac{\partial}{\sim}$ & $\stackrel{2}{\sim}$ & 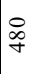 & $\hat{\mathrm{m}}$ & กี & 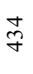 & $\begin{array}{l}\infty \\
\infty \\
m\end{array}$ & $\begin{array}{l}n \\
\infty \\
m\end{array}$ & $\underset{\sim}{ \pm}$ & 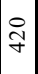 \\
\hline DGPs & & \begin{tabular}{l}
+ \\
\hdashline \\
0
\end{tabular} & $\begin{array}{l}n \\
\infty \\
0 \\
0\end{array}$ & $\begin{array}{l}0 \\
\infty \\
\sim \\
0\end{array}$ & $\begin{array}{l}\infty \\
\infty \\
1 \\
0\end{array}$ & \begin{tabular}{l}
$a$ \\
\multirow{2}{a}{} \\
0
\end{tabular} & \begin{tabular}{l}
+ \\
\hdashline \\
0
\end{tabular} & $\begin{array}{l}n \\
\infty \\
0 \\
0\end{array}$ & \begin{tabular}{l}
$a$ \\
\multirow{2}{0}{} \\
0
\end{tabular} & ָָ & $\begin{array}{l}0 \\
\\
0 \\
0\end{array}$ & $\begin{array}{l}\infty \\
\stackrel{1}{\sim} \\
0\end{array}$ & 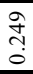 & 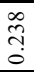 & ָิ & $\frac{0}{\pi}$ & 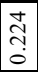 & 吕 & $\frac{m}{a}$ & $\begin{array}{l}\infty \\
\stackrel{+}{0} \\
0\end{array}$ & 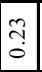 & $\stackrel{\Xi}{\sim}$ & 仓े & $\stackrel{0}{\pi}$ & च̃ & 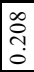 & \begin{tabular}{|l|}
$\infty$ \\
$\stackrel{\infty}{1}$ \\
0 \\
\end{tabular} & $\frac{n}{\sim}$ & $\vec{n}$ \\
\hline Ev (lx) & & 0 & I & \begin{tabular}{l}
\multirow{J}{*}{} \\
$\infty$
\end{tabular} & on & $\stackrel{\sim}{\exists}$ & 0 & 0 & $\frac{0}{1}$ & $\frac{a}{\infty}$ & $\frac{0}{a}$ & 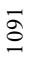 & $\stackrel{m}{\stackrel{2}{0}}$ & $\vec{\infty}$ & હે & $\frac{7}{6}$ & $\hat{6}$ & $\stackrel{ }{I}$ & à & 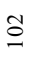 & $\stackrel{m}{n}$ & $\stackrel{8}{\circ}$ & $\stackrel{\infty}{\mathfrak{r}}$ & in & $\stackrel{+}{\dot{q}}$ & s & $\begin{array}{ll} \\
\\
\end{array}$ & $\frac{m}{6}$ & 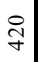 \\
\hline DGPs & & $\begin{array}{l}\frac{1}{\infty} \\
\end{array}$ & $\frac{n}{\infty}$ & 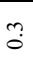 & $\begin{array}{l}1 \\
0 \\
\text { ? } \\
0\end{array}$ & $\underset{N}{N}$ & \begin{tabular}{l}
+ \\
\hdashline \\
0
\end{tabular} & $\begin{array}{l}n \\
\infty \\
0\end{array}$ & 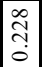 & $\stackrel{n}{n}$ & ָे & กิ & ñ & ले & 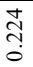 & ป̃ & 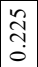 & $\frac{n}{2}$ & $\frac{9}{0}$ & $\frac{a}{0}$ & $\stackrel{m}{\dddot{m}}$ & $\frac{n}{\sim}$ & 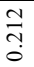 & $\frac{a}{\sim}$ & 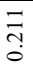 & $\begin{array}{l}\text { oे } \\
\text { ஸे } \\
0\end{array}$ & $\frac{m}{\sim}$ & 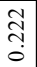 & సָ. \\
\hline Ev (lx) & \multirow{2}{*}{ 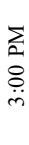 } & 6 & ป & $\stackrel{\infty}{=}$ & $\stackrel{\stackrel{ }{+}}{\text { - }}$ & $\stackrel{n}{=}$ & $\infty$ & $m$ & $\begin{array}{l}\underset{\sim}{v} \\
\stackrel{2}{*}\end{array}$ & $\begin{array}{l}\bullet \\
\stackrel{+}{+}\end{array}$ & $\vec{n}$ & $\stackrel{n}{\infty}$ & $\stackrel{\text { I }}{\text { I }}$ & $\stackrel{\text { ळे }}{\forall}$ & $\vec{m}$ & ભે & $\stackrel{N}{N}$ & $\frac{n}{\sim}$ & 吉 & $i$ & $\begin{array}{l}0 \\
m \\
m\end{array}$ & $\stackrel{\widetilde{N}}{\sim}$ & $\underline{\sigma}$ & $\infty$ & $\hat{\sigma}$ & $\cong$ & $\stackrel{2}{I}$ & \& & \& \\
\hline DGPs & & $\begin{array}{l}\dot{\infty} \\
\stackrel{0}{0}\end{array}$ & $\frac{n}{\infty}$ & in & $\begin{array}{l}\overrightarrow{0} \\
\text { Na } \\
0\end{array}$ & $\begin{array}{l}\tilde{n} \\
\sim \\
0\end{array}$ & $\stackrel{\nexists}{Ð}$ & $\begin{array}{l}\infty \\
\infty \\
0\end{array}$ & $\stackrel{\sim}{0}$ & $\frac{m}{\sim}$ & $\frac{0}{\sim}$ & $\stackrel{ \pm}{\stackrel{ \pm}{~}}$ & $\frac{N}{\sim}$ & $\begin{array}{l}\text { च̣ } \\
\text { o }\end{array}$ & 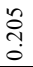 & $\frac{\stackrel{\partial}{\circ}}{\circ}$ & $\begin{array}{l}\overline{0} \\
\text { ஸे } \\
0\end{array}$ & $\frac{\hat{a}}{0}$ & $\frac{\hat{\sigma}}{0}$ & \begin{tabular}{l}
$\infty$ \\
$\infty$ \\
\hdashline \\
0
\end{tabular} & \begin{tabular}{|l|}
$n$ \\
0 \\
\\
0 \\
\end{tabular} & $\frac{\infty}{\stackrel{0}{0}}$ & $\frac{ \pm}{\stackrel{0}{0}}$ & $\frac{n}{2}$ & $\stackrel{\circ}{\circ}$ & $\frac{2}{2}$ & $\frac{n}{2}$ & $\frac{m}{0}$ & $\frac{\tilde{\sigma}}{0}$ \\
\hline
\end{tabular}

From the above result, it is clear that most of DGPs values $(99.9 \%)$ for summer and winter were below 0.35 , which indicated acceptable DGPs values using the mashrabiya device during the entire day. 


\subsection{Simulation}

\subsubsection{Average illuminance}

The average illuminance for simulated room without a mashrabiya was calculated using equation (1). Table 5 has shown this average illuminance for both summer and winter.

Table 5: Average illuminance (lx) without mashrabiya in summer and winter.

\begin{tabular}{|c|c|c|}
\hline \multirow{2}{*}{ Time } & Average illuminance without mashrabiya (lx) from simulation (lx) \\
\cline { 2 - 3 } & Winter & Summer \\
\hline 9:00 am & 1518 & 929 \\
\hline Solar noon & 3142 & 1158 \\
\hline 3:00 pm & 1652 & 848 \\
\hline
\end{tabular}

4.2.2 Daylight uniformity ratio

The uniformity of the daylight distribution in the simulated space was calculated using equation (3). Results indicate very low uniformity in the space without mashrabiya during winter. In summer, the ratio was very low at 3:00 pm; however, for 9:00 am and noon, the results were equal to the recommended ratio of 0.4 . Table 6 shows;

Table 6: Uniformity ratio without mashrabiya in summer and winter.

\begin{tabular}{|c|c|c|}
\hline \multirow{2}{*}{ Time } & Uniformity Ratio without mashrabiya (lx) \\
\cline { 2 - 3 } & Winter & Summer \\
\hline 9:00 am & 0.4 & 0.1 \\
\hline Solar noon & 0.4 & 0.1 \\
\hline 3:00 pm & 0.1 & 0.1 \\
\hline
\end{tabular}

\subsubsection{Daylight glare probability index}

A simplified daylight glare index (DGIs) was calculated for each point in the studied space by simulation for the specified times and seasons similar to the actual measurements but without the mashrabiya (Tables 7 and 8) using eqn (4) [16], [17].

Table 7: Simplified daylight glare probability (DGPs) for summer at 9:00 am, noon, and 3:00 pm for simulation readings.

\begin{tabular}{|c|c|c|c|c|c|c|c|c|c|c|c|c|c|c|c|c|c|c|c|c|c|c|c|c|c|c|c|c|c|}
\hline $\begin{array}{l}\text { Measurement } \\
\text { points }\end{array}$ & & 1 & 2 & 3 & 4 & 5 & 6 & 7 & 8 & 9 & 10 & 11 & 12 & 13 & 14 & 15 & 16 & 17 & 18 & 19 & 20 & 21 & 22 & 23 & 24 & 25 & 26 & 27 & 28 \\
\hline Ev (lx) & $\Sigma$ & $\begin{array}{l}\text { i } \\
\text { d } \\
\text { d }\end{array}$ & 용 & ㄱ. & $\frac{d}{\stackrel{a}{2}}$ & $\vec{\nabla}$ & 古 & $\bar{\sigma}$ & $\stackrel{\circ}{\curvearrowright}$ & $\mid \begin{array}{l}\infty \\
\Sigma \\
\Sigma\end{array}$ & $\begin{array}{l}0 \\
\infty \\
\infty \\
-1\end{array}$ & $\stackrel{尺}{\stackrel{\overbrace{}}{\curvearrowright}}$ & $\stackrel{2}{\text { ปे }}$ & $\begin{array}{l}n \\
0 \\
2 \\
2\end{array}$ & $\begin{array}{l}0 \\
\infty \\
=\end{array}$ & $\stackrel{2}{2}$ & 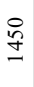 & \begin{tabular}{l}
8 \\
0 \\
\hdashline \\
-1
\end{tabular} & $\stackrel{n}{\circ}$ & 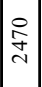 & $\stackrel{\infty}{\stackrel{\infty}{\sim}}$ & 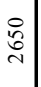 & $\vec{\sim}$ & $\vec{n}$ & 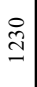 & $\begin{array}{l}a \\
\approx\end{array}$ & $\frac{m}{N}$ & $\begin{array}{l}n \\
n \\
\sim\end{array}$ & $\begin{array}{l}\infty \\
\stackrel{+}{\sim}\end{array}$ \\
\hline DGPs & & $\hat{m}$ & $\frac{m}{n}$ & \begin{tabular}{|l|}
$m$ \\
$m$ \\
0 \\
\end{tabular} & ?ై & $\stackrel{m}{m}$ & $\begin{array}{l}0 \\
\tilde{m} \\
\tilde{o} \\
0\end{array}$ & $\begin{array}{l}\infty \\
\sim \\
\sim \\
0\end{array}$ & $\begin{array}{l}n \\
\tilde{n} \\
0\end{array}$ & 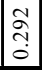 & \begin{tabular}{|l|} 
\\
\multirow{2}{*}{} \\
0 \\
0
\end{tabular} & $\begin{array}{ll}m \\
m \\
0 \\
\end{array}$ & กิ & $\begin{array}{l}\vec{m} \\
? \\
0\end{array}$ & \begin{tabular}{|l|}
$\infty$ \\
$\sim$ \\
$n$ \\
0 \\
\end{tabular} & $\begin{array}{l}5 \\
0 \\
0 \\
0 \\
0\end{array}$ & సุ & $\begin{array}{l}\vec{\infty} \\
\sim \\
0 \\
\end{array}$ & $\begin{array}{c}1 \\
m \\
0\end{array}$ & $\begin{array}{l}\infty \\
m \\
n \\
0 \\
\end{array}$ & \begin{tabular}{|l|}
$n$ \\
$n$ \\
$n$ \\
0
\end{tabular} & 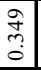 & \begin{tabular}{|l|}
\multirow{2}{*}{} \\
\multirow{2}{*}{} \\
0 \\
\end{tabular} & \begin{tabular}{|l|}
\multirow{2}{*}{} \\
\\
0 \\
\end{tabular} & 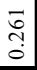 & $\begin{array}{l}\vec{n} \\
\tilde{n} \\
0\end{array}$ & \begin{tabular}{|l|}
$n$ \\
$n$ \\
0 \\
0
\end{tabular} & $\stackrel{m}{m}$ & $\begin{array}{l}\infty \\
m \\
n \\
0\end{array}$ \\
\hline Ev (lx) & & $\begin{array}{l}\infty \\
\infty \\
m\end{array}$ & N & $\begin{array}{l}0 \\
m \\
m\end{array}$ & $\begin{array}{l}\text { N } \\
\text { n } \\
m\end{array}$ & $\begin{array}{l}n \\
n \\
n \\
n\end{array}$ & $\begin{array}{l}\circ \\
2 \\
2\end{array}$ & 을 & $\stackrel{0}{n}$ & $\frac{m}{m}$ & \begin{tabular}{|l|}
$n$ \\
0 \\
$n$ \\
$m$
\end{tabular} & $\begin{array}{l}n \\
\infty \\
2 \\
m\end{array}$ & 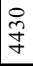 & $\frac{0}{n}$ & $\begin{array}{l}\Omega \\
\\
\end{array}$ & $\stackrel{\infty}{\stackrel{\infty}{m}}$ & 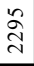 & $\begin{array}{l}\stackrel{8}{ } \\
\stackrel{+}{\sim}\end{array}$ & $\begin{array}{l}n \\
\text { m } \\
m\end{array}$ & $\begin{array}{l}n \\
\infty \\
2 \\
2\end{array}$ & $\begin{array}{l}\tilde{N} \\
\text { nे } \\
\end{array}$ & $\underset{\stackrel{\sim}{\sim}}{\stackrel{\sim}{\sim}}$ & $\begin{array}{l}\widetilde{N} \\
\stackrel{J}{\sim}\end{array}$ & $\begin{array}{l}0 \\
\infty \\
2 \\
-\end{array}$ & $\begin{array}{l}\text { రু } \\
\text { - }\end{array}$ & $\begin{array}{l}0 \\
+ \\
n \\
m\end{array}$ & \begin{tabular}{c}
0 \\
$\stackrel{0}{0}$ \\
\multirow{\sigma}{*}{}
\end{tabular} & $\begin{array}{l}8 \\
\end{array}$ & 过 \\
\hline DGPs & z & $\stackrel{\sim}{\stackrel{N}{*}} \underset{0}{\circ}$ & $\begin{array}{l}\vec{\alpha} \\
\tilde{o}\end{array}$ & $\begin{array}{l}\stackrel{8}{\alpha} \\
\text { ஸे } \\
0\end{array}$ & @o & $\begin{array}{l}\exists \\
\Xi \\
\stackrel{0}{0}\end{array}$ & $\begin{array}{l}0 \\
\stackrel{\oplus}{*} \\
\dot{+}\end{array}$ & 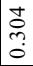 & 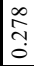 & $\begin{array}{l}\stackrel{2}{\circ} \\
\text { ஸे } \\
0\end{array}$ & 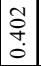 & $\begin{array}{l}N \\
\stackrel{v}{+} \\
\dot{0}\end{array}$ & \begin{tabular}{l}
0 \\
0 \\
\multirow{0}{*}{} \\
0
\end{tabular} & $\begin{array}{l}n \\
0 \\
0 \\
0 \\
0\end{array}$ & $\begin{array}{l}0 \\
\sim \\
\sim \\
0 \\
0\end{array}$ & $\begin{array}{l}0 \\
\infty \\
\\
0\end{array}$ & 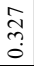 & 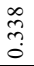 & $\begin{array}{l}\stackrel{2}{2} \\
\text { m. } \\
0\end{array}$ & $\mid \begin{array}{c}\tilde{N} \\
\tilde{\sigma} \\
\dot{0}\end{array}$ & \begin{tabular}{|c|}
$\infty$ \\
$\stackrel{N}{\sim}$ \\
$\stackrel{+}{0}$
\end{tabular} & $\begin{array}{l}\stackrel{0}{0} \\
\stackrel{+}{*} \\
0\end{array}$ & $\begin{array}{l}n \\
m \\
n \\
0\end{array}$ & \begin{tabular}{|l|}
$\infty$ \\
0 \\
0 \\
$\tilde{0}$ \\
0
\end{tabular} & $\begin{array}{l}0 \\
\stackrel{0}{0} \\
0\end{array}$ & $\mid \begin{array}{l}n \\
o \\
+ \\
0 \\
0\end{array}$ & $\begin{array}{l}0 \\
0 \\
0 \\
0 \\
0\end{array}$ & 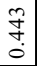 & $\begin{array}{l}n \\
\tilde{\sigma} \\
0 \\
0\end{array}$ \\
\hline Ev (lx) & $\Sigma$ & $\begin{array}{l}n \\
\stackrel{5}{n} \\
\sim\end{array}$ & $\frac{\dot{n}}{\sim}$ & $\stackrel{m}{\sim}$ & $\stackrel{\infty}{\stackrel{\infty}{\sim}}$ & $\begin{array}{l}\infty \\
\stackrel{\infty}{+} \\
\sim\end{array}$ & $\begin{array}{l} \pm \\
\tilde{n} \\
\sim\end{array}$ & $\stackrel{a}{\beth}$ & $\underset{\infty}{+}$ & $\stackrel{\circ}{\triangleright}$ & 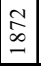 & $\begin{array}{l}\bar{Z} \\
\bar{v}\end{array}$ & $\begin{array}{l}\stackrel{0}{2} \\
\text { } \\
\stackrel{\sim}{v}\end{array}$ & 今ે & 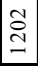 & $\underset{\sim}{\vec{J}}$ & $\begin{array}{l}\infty \\
\stackrel{\infty}{ \pm} \\
\end{array}$ & $\begin{array}{l}\infty \\
\infty \\
\\
-\end{array}$ & $\stackrel{\circ}{\circ}$ & $\begin{array}{l} \\
\\
\sim \\
\sim \\
\end{array}$ & \begin{tabular}{|l|} 
\\
\\
\end{tabular} & 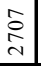 & \begin{tabular}{|l|} 
\\
0 \\
0 \\
-1 \\
\end{tabular} & \begin{tabular}{|l|} 
\\
$\infty$ \\
N \\
\end{tabular} & $\begin{array}{l}\stackrel{8}{0} \\
\stackrel{-}{1}\end{array}$ & $\begin{array}{l} \pm \\
\text { N }\end{array}$ & $\begin{array}{l} \\
\\
\end{array}$ & 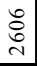 & $\begin{array}{l}0 \\
i n \\
i\end{array}$ \\
\hline DGPs & 암 & 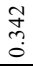 & $\frac{\infty}{m}$ & $\begin{array}{c}n \\
m \\
0 \\
0\end{array}$ & $\begin{array}{l}\stackrel{+}{d} \\
\text { ?n } \\
0\end{array}$ & $\stackrel{m}{m}$ & \begin{tabular}{c}
$\mathcal{Y}$ \\
\multirow{y}{*}{} \\
$\tilde{0}$ \\
\end{tabular} & ֻ̊ & స̃ & $\begin{array}{l}n \\
\text { กิ } \\
0\end{array}$ & $\begin{array}{l}n \\
0\end{array}$ & $\frac{n}{n}$ & 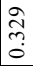 & $\begin{array}{l}m \\
\tilde{m} \\
0\end{array}$ & 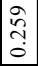 & $\begin{array}{c}\exists \\
\tilde{m} \\
0\end{array}$ & $\begin{array}{l}\stackrel{0}{~} \\
\text { N̦} \\
0\end{array}$ & 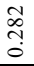 & 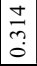 & $\begin{array}{l}+ \\
\tilde{m} \\
0\end{array}$ & \begin{tabular}{|l|} 
\\
$\tilde{n}$ \\
$\tilde{n}$ \\
0
\end{tabular} & $\begin{array}{l}N \\
n \\
n \\
0\end{array}$ & $\begin{array}{l} \\
\infty \\
\\
0\end{array}$ & 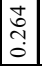 & $\begin{array}{l}\text { Ĵ } \\
\text { N̦ } \\
0\end{array}$ & $\begin{array}{l}\tilde{N} \\
\tilde{N} \\
0\end{array}$ & \begin{tabular}{|c|} 
\\
$n$ \\
$n$ \\
0 \\
\end{tabular} & $\begin{array}{l}0 \\
\stackrel{0}{*} \\
m \\
0\end{array}$ & 吉 \\
\hline
\end{tabular}


Table 8: Simplified daylight glare probability (DGPs) for winter at 9:00 am, noon, and 3:00 pm for simulation readings.

\begin{tabular}{|c|c|c|c|c|c|c|c|c|c|c|c|c|c|c|c|c|c|c|c|c|c|c|c|c|c|c|c|c|c|}
\hline $\begin{array}{l}\text { Measure } \\
\text { points }\end{array}$ & & 1 & 2 & 3 & 4 & 5 & 6 & 7 & 8 & 9 & 10 & 11 & 12 & 13 & 14 & 15 & 16 & 17 & 18 & 19 & 20 & 21 & 22 & 23 & 24 & 25 & 26 & 27 & 28 \\
\hline$E_{v}$ & $\Sigma$ & 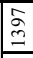 & $\bar{\Xi}$ & $\frac{m}{\stackrel{N}{a}}$ & $\frac{\infty}{\underline{\Xi}}$ & \begin{tabular}{|l|}
\multirow{2}{*}{} \\
$m$ \\
\end{tabular} & $\begin{array}{l}3 \\
0 \\
\\
\end{array}$ & 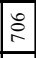 & \begin{tabular}{|l|}
\multirow{2}{*}{} \\
\\
\end{tabular} & \begin{tabular}{|l|}
\multirow{2}{*}{} \\
İ \\
\end{tabular} & $\begin{array}{l} \\
\infty \\
0 \\
\end{array}$ & $\stackrel{ \pm}{\beth}$ & $\mid \begin{array}{l}\infty \\
- \\
-1\end{array}$ & 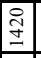 & $\mathbb{N}$ & \begin{tabular}{l|}
$\stackrel{\infty}{=}$ \\
\end{tabular} & \begin{tabular}{|l|} 
\\
0 \\
\end{tabular} & $\begin{array}{l}n \\
\tilde{\alpha}\end{array}$ & 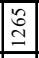 & ñ & $\underline{n}$ & 点 & $\frac{1}{2}$ & $\stackrel{i}{2}$ & I & \begin{tabular}{|l|}
\multirow{2}{*}{} \\
2 \\
\end{tabular} & 吉 & \begin{tabular}{|l|}
$\infty$ \\
0 \\
\\
\end{tabular} & 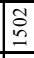 \\
\hline DGP & 8 & $\vec{\nabla}$ & 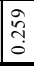 & 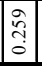 & 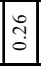 & \begin{tabular}{l|} 
\\
$\infty$ \\
0 \\
\\
0 \\
\end{tabular} & $\stackrel{n}{\sim}$ & $\stackrel{\infty}{\sim}$ & \begin{tabular}{|l|}
\multirow{2}{*}{} \\
\multirow{2}{*}{} \\
\end{tabular} & \begin{tabular}{|l|}
\multirow{2}{*}{} \\
\\
0 \\
\end{tabular} & 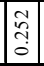 & $\overrightarrow{0}$ & ஸ̂. & \begin{tabular}{|l|}
\multirow{N}{*}{} \\
\multirow{2}{*}{} \\
\end{tabular} & \begin{tabular}{|l|}
\multirow{2}{*}{} \\
ஸे \\
\end{tabular} & 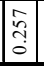 & \begin{tabular}{|c|}
\multirow{2}{*}{} \\
\multirow{2}{*}{} \\
0 \\
\end{tabular} & \begin{tabular}{|c|}
\multirow{2}{*}{} \\
ปै \\
\end{tabular} & \begin{tabular}{|l|} 
\\
\multirow{2}{*}{} \\
$\tilde{0}$ \\
\end{tabular} & \begin{tabular}{l}
$\infty$ \\
\multirow{2}{*}{} \\
0
\end{tabular} & \begin{tabular}{|l|}
\multirow{2}{*}{} \\
\\
0 \\
\end{tabular} & $\hat{\infty}$ & 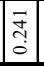 & 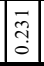 & \begin{tabular}{|c|}
\multirow{2}{*}{} \\
\end{tabular} & 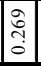 & $\approx$ & $\infty$ & సิ \\
\hline$E_{v}(1$ & 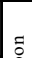 & 过 & 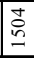 & 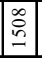 & $\begin{array}{l}\infty \\
\stackrel{2}{2} \\
\end{array}$ & $\stackrel{2}{\Xi}$ & 2 & 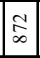 & \begin{tabular}{|l|} 
\\
$\infty$ \\
$\infty$ \\
\end{tabular} & \begin{tabular}{|l|}
\multirow{2}{*}{} \\
$\stackrel{-}{-}$ \\
\end{tabular} & $\begin{array}{l}0 \\
n \\
m \\
-\end{array}$ & in & $\frac{n}{5}$ & $\stackrel{n}{\check{2}}$ & $\stackrel{\circ}{\infty}$ & 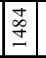 & \& & $\stackrel{\cong}{\Xi}$ & 吾 & $\begin{array}{l}\infty \\
\infty \\
\infty \\
\end{array}$ & \begin{tabular}{|l|} 
\\
$\infty$ \\
$\infty$ \\
- \\
\end{tabular} & $\because 2$ & t & 吕 & $\begin{array}{l} \\
\text { ñ }\end{array}$ & \begin{tabular}{|l|} 
\\
0 \\
0 \\
0 \\
\end{tabular} & 8 & $\vec{\infty}$ & 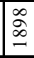 \\
\hline$D$ & & 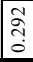 & \begin{tabular}{|l|}
\multirow{2}{*}{} \\
\multirow{2}{*}{} \\
0 \\
\end{tabular} & \begin{tabular}{|l|}
\multirow{2}{*}{} \\
\multirow{2}{*}{} \\
0 \\
\end{tabular} & \begin{tabular}{|l|} 
\\
$\infty$ \\
1 \\
0 \\
\end{tabular} & సે & $\begin{array}{l}\text { กे } \\
\text { సุ. }\end{array}$ & \begin{tabular}{|c|}
$\infty$ \\
\multirow{2}{n}{} \\
0 \\
\end{tabular} & 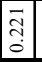 & \begin{tabular}{|l|}
\multirow{0}{*}{} \\
\\
0 \\
\end{tabular} & 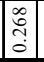 & 文 & \begin{tabular}{|l|}
$\vec{\sigma}$ \\
సे \\
\end{tabular} & 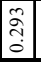 & 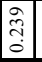 & \begin{tabular}{|l|}
\multirow{2}{*}{} \\
\\
0 \\
\end{tabular} & \begin{tabular}{|c|}
\multirow{2}{*}{} \\
กุ. \\
\end{tabular} & 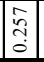 & \begin{tabular}{|l|}
\multirow{2}{*}{} \\
\\
\\
\end{tabular} & \begin{tabular}{|c|}
0 \\
\\
0 \\
0
\end{tabular} & \begin{tabular}{|l|}
\multirow{2}{*}{} \\
กे
\end{tabular} & g & \begin{tabular}{|l|} 
\\
\end{tabular} & \begin{tabular}{|l|}
\multirow{f}{*}{} \\
\multirow{2}{*}{} \\
0
\end{tabular} & \begin{tabular}{|c|}
\multirow{2}{*}{} \\
\multirow{2}{*}{} \\
0 \\
\end{tabular} & \begin{tabular}{|l|}
\multirow{2}{*}{} \\
\multirow{2}{*}{} \\
0 \\
\end{tabular} & \begin{tabular}{|l|}
$\frac{n}{3}$ \\
$\vdots$ \\
0
\end{tabular} & 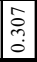 & \begin{tabular}{|l|}
\multirow{2}{*}{} \\
$\tilde{n}$ \\
0 \\
\end{tabular} \\
\hline & 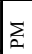 & I) & 负 & హ్ & $\frac{\infty}{0}$ & $\begin{array}{l}\hat{\Xi} \\
\varrho\end{array}$ & $\stackrel{g}{\Xi}$ & $\begin{array}{l}n \\
n \\
n\end{array}$ & 尽 & $\begin{array}{l}\text { त्र } \\
\infty\end{array}$ & $\begin{array}{l}\underset{D}{\infty} \\
\infty\end{array}$ & $\infty$ & $\stackrel{8}{\circ}$ & $\begin{array}{l}\stackrel{\Xi}{\Xi} \\
\end{array}$ & $\begin{array}{l}n \\
i n \\
\end{array}$ & $\begin{array}{l}3 \\
\infty \\
\sigma\end{array}$ & 8. & $F$ & 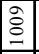 & $\begin{array}{l} \\
\stackrel{2}{\simeq} \\
\end{array}$ & $\stackrel{\Omega}{\Xi}$ & $\begin{array}{l}\overrightarrow{0} \\
-\end{array}$ & $\stackrel{n}{\circ}$ & \begin{tabular}{|l|} 
\\
\end{tabular} & $\begin{array}{l}2 \\
\text { in }\end{array}$ & $\stackrel{I}{\Xi}$ & \begin{tabular}{|l|}
\multirow{2}{*}{} \\
ले \\
\end{tabular} & 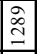 & $\hat{\imath}$ \\
\hline GPs & & \begin{tabular}{|l} 
\\
岁
\end{tabular} & \begin{tabular}{|l}
\multirow{2}{*}{} \\
$\stackrel{2}{0}$ \\
\end{tabular} & \begin{tabular}{|l|}
\multirow{2}{*}{} \\
$\stackrel{2}{ }$ \\
\\
\end{tabular} & \begin{tabular}{|l|}
\multirow{2}{*}{} \\
\multirow{2}{0}{} \\
\end{tabular} & 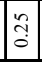 & $n$ & $\frac{\infty}{2}$ & \begin{tabular}{|l|}
\multirow{2}{*}{} \\
\multirow{2}{*}{} \\
\end{tabular} & \begin{tabular}{|l|}
$n$ \\
\\
0 \\
\end{tabular} & \begin{tabular}{|c|}
$\infty$ \\
\multirow{n}{*}{} \\
0 \\
\end{tabular} & 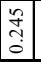 & $\begin{array}{l}\text { N } \\
\text { กิ } \\
0\end{array}$ & 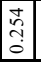 & 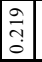 & 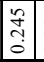 & 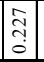 & $\tilde{\jmath}$ & \begin{tabular}{|l|}
\multirow{5}{*}{} \\
\\
0
\end{tabular} & 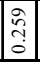 & \begin{tabular}{|l|}
$\infty$ \\
\\
$\tilde{c}$ \\
\end{tabular} & \begin{tabular}{|l|}
$n$ \\
0 \\
2 \\
0 \\
\end{tabular} & 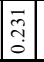 & 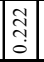 & 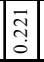 & \begin{tabular}{|c|}
\multirow{2}{*}{} \\
\multirow{2}{*}{} \\
0 \\
\end{tabular} & 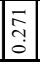 & \begin{tabular}{|l|}
\multirow{2}{*}{} \\
Ț \\
0 \\
\end{tabular} & i \\
\hline
\end{tabular}

Summer results of daylight glare probability at 9:00 am and 3:00 pm have shown that there were no problems of glare. Results show that $28 \%$ of the reading points were located in the best class, which were $\leq 0.35$. However, $35.7 \%$ were located in good class $\leq 0.42,25 \%$ was located in the reasonable class $\leq 0.45$, and $10.7 \%$ were considered disturbing values. Winter results show that all readings located in the best class as all reading points were $\leq 0.35$.

\subsection{Comparison of the actual and simulation results}

4.1.1 Average illuminance of the actual and simulated room

Table 9 has shown a comparison between the average illuminance levels for the studied room with mashrabiya, without mashrabiya, and the recommended lighting level for the room in the house. The averages for both summer and winter were taken at 9:00 am, solar noon, and 3:00 pm.

Table 9: Comparison of average illuminance (lx) in summer and winters.

\begin{tabular}{|c|c|c|c|c|c|}
\hline Time & \multicolumn{2}{|c|}{$\begin{array}{c}\text { Average illuminance } \\
\text { with mashrabiya in lx } \\
\text { (Eavg) }\end{array}$} & \multicolumn{2}{c|}{$\begin{array}{c}\text { Average illuminance } \\
\text { without mashrabiya }\end{array}$} & $\begin{array}{c}\text { Recommended illuminance } \\
\text { in lx for residence } \\
\text { (AS/NZS1680.2, 1990) }\end{array}$ \\
\hline & Winter & Summer & Winter & Summer & \\
\hline 9:00 am & 159 & 235 & 929 & 1518 & $160-240$ \\
\hline Solar noon & 180 & 218 & 1158 & 3142 & $160-240$ \\
\hline 3:00 pm & 99 & 193 & 848 & 1652 & $160-240$ \\
\hline
\end{tabular}

4.1.2 Uniformity ratio (minimum to average) of the actual and simulated rooms

This calculation was carried out with the results of the actual readings and the simulation readings. Figs 11 and 12 have shown a comparison of the uniformity (minimum to average ratio) of the room with and without the mashrabiya, in both summer and winter and at three different times.

The results from Figs 11 and 12 have shown that during summer, the uniformity ratio, which is 0.4 , is equal in both conditions (i.e., with and without mashrabiya). For other times of the day, the uniformity without mashrabiya shows very low values in comparison with results with mashrabiya. In winter, the ratios for the three times are higher for the opening with mashrabiya than the one time without mashrabiya. Figs 13 and 14 have shown the daylight in the space for the actual room with the mashrabiya and the simulation for the same room without the mashrabiya. 


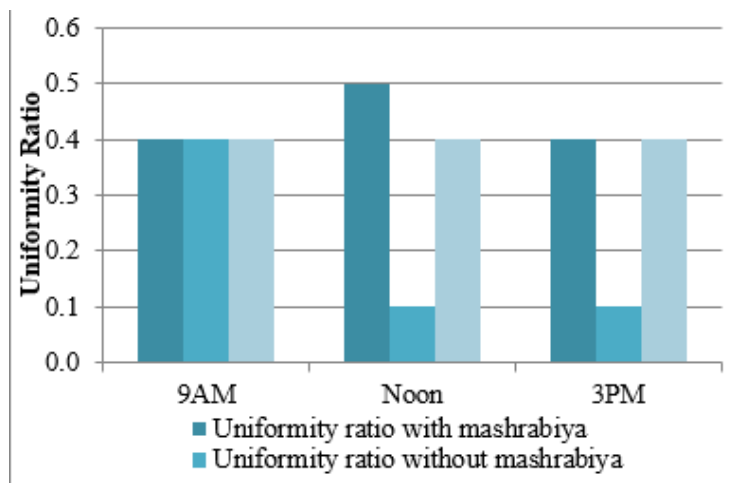

Figure 11: Comparison between uniformity ratio in summer with mashrabiya, without mashrabiya and recommended uniformity ratio.

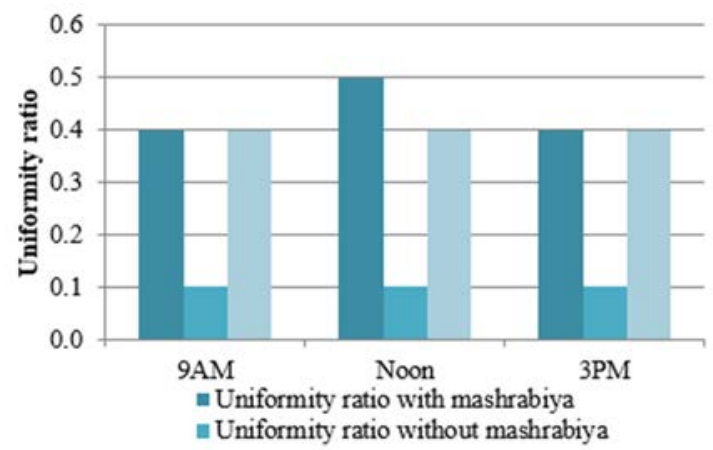

Figure 12: Comparison between uniformity ratio in winter with mashrabiya, without mashrabiya and the recommended uniformity ratio.

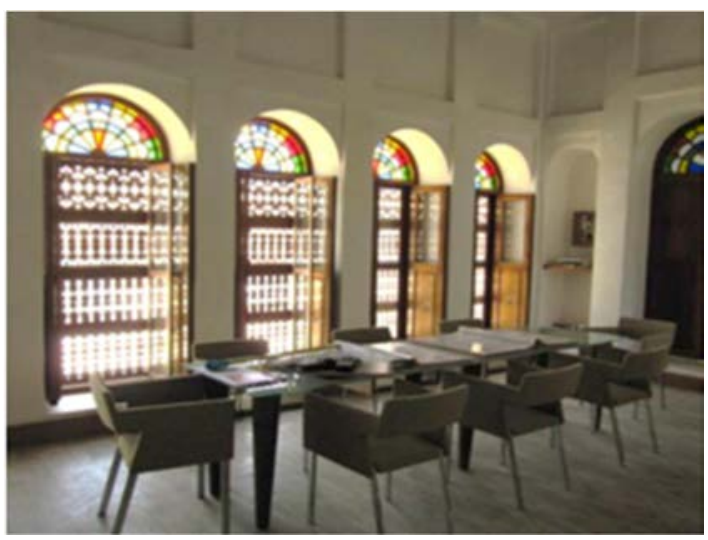

Figure 13: An actual picture of the room at $9.00 \mathrm{am}$. 


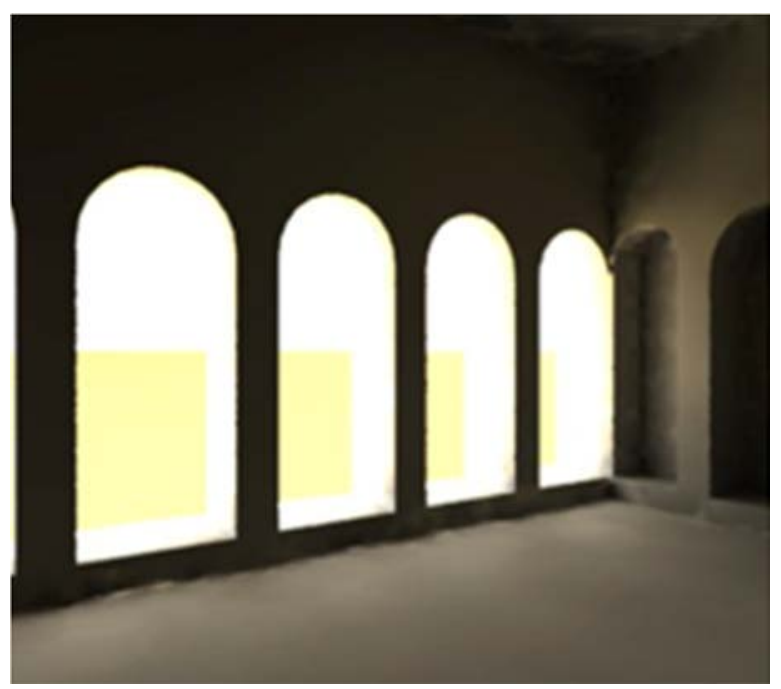

Figure 14: A ray-traced image of the room without mashrabiya at 9.00 am (Prepared using Ecotect and Desktop Radiance).

\subsubsection{Daylight glare probability index of the actual and simulated rooms}

Comparison of the daylight glare probability results for the actual room and simulated room have shown that although the vertical illuminance for the room without mashrabiya was much higher than the vertical illuminance with mashrabiya. Moreover, no glare problems were indicated for the two cases. This excludes the simulated room at noontime in summer, as the result was higher than all other readings. This may require window treatment to decrease the effect of the glare for noontime. The reason that there is no noticeable glare problem for the room without any shading device or mashrabiya may be that the room is facing north in the northern hemisphere, where no direct sunlight accesses the space. As discussed earlier, the mashrabiya was designed in the past mainly to achieve visual privacy, but also to allow cross ventilation and minimise sharp sunlight within the space [1], [2].

\section{CONCLUSION}

The study results have concluded that the mashrabiya in this case can provide the level of illuminance recommended for residential activities and can provide better uniformity than can the space without mashrabiya. However, it is preferable to improve the design of the mashrabiya to enhance the uniformity of daylight in the space. Another challenge regarding the performance of the mashrabiya device concerns the chromaticity of light in the space as the CCT calculation for all the measured points were below the accepted level. On the other hand, a study of the daylight glare index showed that the mashrabiya device could reduce the effect of glare in the whole space.

As Bahrain is a sunny country, it is preferable to have shading devices to minimise the glare and the amount of direct sunlight entering the space. However, it cannot be said that the mashrabiya was $100 \%$ successful at this because of the mentioned challenges. Therefore, if this mashrabiya were redesigned or modified to consider uniformity and the chromaticity of light in the space, it could demonstrate a more successful performance and better daylighting. 


\section{ACKNOWLEDGEMENT}

The authors are very thankful to all the associated personnel in any reference that contributed in/for the purpose of this research.

\section{REFERENCES}

[1] Aljuboori, U.A., Light in Islamic architecture: Natural light configurations in contemporary mosques. Presented at the International Scientific Conference: Art in Islamic Thought, Jordan, 2012.

[2] El-Shorbagy, A., Traditional Islamic-Arab house: Vocabulary and syntax. International Journal of Civil and Environmental Engineering IJCEE-IJENS, 10, pp. 15-20, 2010.

[3] Madden, E.H., Some characteristics of Islamic Art. The Journal of Aesthetics and Art Criticism, 33, pp. 423-430, 1975.

[4] Olgyay, V., Bioclimatic evaluation method for architectural application. Biometeorology, Pergamon, pp. 246-261, 1962.

[5] Thateenaranon, P., Amornkitbamrung, M., Hirunlabh, J., Khedari, J. \& Waewsak, J., Full-scale field investigation of a bio-climatic house under Thailand tropical climate. Building and Environment, 126, pp. 54-67, 2017.

[6] Okello, D., Concept and techniques: sustainable design techniques in the Langi traditional architecture. A case study of the Langi traditional housing. Doctoral dissertation, Makerere University, 2017.

[7] Alnaser, N.W. \& Flanagan, R., The need of sustainable buildings construction in the Kingdom of Bahrain. Building and Environment, 42, pp. 495-506, 2017.

[8] Alnaser, W.E. \& Merzaa, M.K., Empirical correlations for the climate data in the Kingdom of Bahrain. Energy, 30, pp. 2818-2830, 2005.

[9] Attili, B.S. \& Abdalla, Y.A.G., Numerical models for the correlation of global solar radiation with meteorological data for Bahrain. Energy Conversion and Management, 34, pp. 1-6, 1993.

[10] Maghrabi, H. \& Mostafa, Z.A., Estimating surface albedo over Saudi Arabia. Renewable Energy, 34, pp. 1607-1610, 2009.

[11] Yousif, R., Abdullah Al Zayed house for Bahraini Press Heritage, 2011. http://notjustaphoto.me/abdullah-al-zayed-house-for-bahraini-press-heritageextension.

[12] Al-Zayed's House, Retrieved from Gulf House Engineering, 2002.

[13] Kensek, K. \& Suk, J.Y., Daylight factor (overcast sky) versus daylight availability (clear sky) in computer-based daylighting simulations. Journal of Creative Sustainable Architecture and Built Environment, 1, pp. 3-14, 2011.

[14] AS/NZS1680.1, Australia Standard: Interior Lighting Part 1: General Principles and Recommendation, Homebush: Standards Australia, 1990.

[15] The Kruith of comfort curve. The perfect light design, 2013. www.osram.com.au/osram_au/Lighting_Design/About_Light/Getting_the_best_poss ible_light_/The_perfect_light_design/index.html.

[16] Suk, J.-Y., Schiler, M. \& Kensek, K., Development of new daylight glare analysis methodology using absolute glare factor and relative glare factor. Energy and Buildings, 64, pp. 113-122, 2013.

[17] Wienold, J., Dynamic daylight glare evaluation. Presented at the Eleventh International IBPSA Conference, Glasgow, Scotland, 2009. 